\title{
Role of Lung Ultrasound and Inferior Vena Cava Diameter in Assessment of Patients with Heart Failure \\ Hussein Abd El-Fattah Mohammed ${ }^{1}$, Mohamed Salah El-Feshawy², Fareed Shawky Basiony ${ }^{1}$ and Mustafa Maged Abd Alla * \\ ${ }^{1}$ Chest diseases department, ${ }^{2}$ Diagnostic Radiology department, Faculty of Medicine, Al-Azhar University, Egypt \\ *Corresponding Author: Dr. Mustafa Maged Abd Alla, Mobile.:01110005021, \\ E-mail: mostafamg_2004@hotmail.com
}

\section{ABSTRACT}

Background: Congestive heart failure (CHF) is a multifaceted clinical condition marked by recurrent episodes of acute decompensation, needing repetitive hospital stays, and re-admissions. The CHF management is still a clinical challenge; choices are built on clinical evaluations of volume status, which are utilized for cardiac filling pressure estimation.

Aims: This study aimed to investigate the role of lung US and Inferior Vena Cava Diameter (IVCD) in assessment of HF-cases.

Patients and Methods: The current work has been performed on $50 \mathrm{HF}$ (heart failure) cases diagnosed in the Chest Department of Al- Azhar University Hospitals from April 2019 to June 2021. Chest radiograph, as a reference for the presence of pulmonary congestion, echocardiography for both systolic and diastolic function assessment and lung U/S. US examinations of the anterolateral chest were done.

Results: A highly significant association was found among inferior Vena Cava-Collapsibility Index (IVC-CI) and Dyspnea Class. A highly significant association was found among IVC-CI and echocardiography. A highly significant association was found among IVC-CI and B lines.

Conclusion: Ultrasound can be utilized as an alternative technique for estimating the intra-vascular volume such as measuring the IVCD and so the caval index. The utilization of pulmonary ultrasound (PU) to evaluate dyspneic cases and those with HF in dissimilar clinical settings raises the sensitivity, specificity, and accuracy of pulmonary congestion diagnosing and prognosis in HF cases.

Keywords: Lung ultrasound, Inferior vena cava, B lines, Heart failure.

\section{INTRODUCTION}

Congestive heart failure (CHF) is a main public health problem. About 5.7 million cases in the U.S.A diagnosed with CHF with a lifetime frequency of 1 in 5 for persons greater than 40-yrs old. HF is the commonest hospital discharge diagnosing, and more Medi-care cash is paid for CHF management than for any other management. Acutely decompensated HF (ADHF) is as well the commonest reason for acute dyspnea between aging cases in the emergency department. While earlier suitable diagnosing and treatment are accompanying reduced mortalities, ADHF is as well the commonest reason of mortality between dyspneic cases admitted to the emergency department (ED) ${ }^{[1]}$.

Variation among cardiac dyspnea and pulmonary dyspnea is conservatively accomplished via blood tests, physical examinations, and $x$-ray of the chest. But, these examinations have moderate accuracies ${ }^{[2]}$.

Echo-cardiography assessment aids clinicians to perform the demarcation, but it needs advanced skills and training. Therefore, many other laboratory investigations were advanced to make a precise demarcation of dyspnea. Brain natriuretic peptide (BNP) was settled and presented into clinical practices for this aim ${ }^{[3]}$.There are 3 points of care US modalities that have possible efficacy in ADHF diagnosing: cardiac US [which gives straight visualizations of ejection fraction (EF)], IVC-US (a non-invasive method of assessing intra-vascular volume status), and lung US (that may notice the existence of interstitial edema. Each of these modalities was utilized lonely in assessment of diagnosing power. However, when utilized lonely, everyone wants the accuracy desired to finally perform the diagnosing between acutely dyspneic cases in ED.

A depressed left ventricular EF existing in chronic HF doesn't specify whether there was an acute decrease in systolic functions, and neither a plethoric IVC nor the existence of interstitial edema is definite to acute decompensated $\mathrm{HF}{ }^{[4]}$

Correspondingly, bedside US was utilized to determine IVCD to discriminate AHF from other reasons of dyspnea. Some previous researches have concluded that BNP and IVCD were associated, proposing that IVCD can be utilized in the discrimination of dyspnea ${ }^{[5]}$.

\section{AIM OF THE WORK}

This study was designed to investigate the role of lung US and IVC Diameter in assessment of HF cases.

\section{PATIENTS AND METHODS}

The current work included $50 \mathrm{HF}$ cases diagnosed in the Chest Department of Al- Azhar University Hospitals from April 2019 to June 2021.

Inclusion criteria: An acute decompensation due to clinically evident pulmonary congestion not requiring emergency treatment.sépiage between 18 \& 65-yearold. 
Exclusion criteria: An event such as atrial fibrillation, severe aortic regurgitation, prosthetic mitral valve or previous mitral repair, pericardial effusion, severe renal failures (creatinine clearances $<25 \mathrm{ml} / \mathrm{min}$ ).

Creatinine clearance: The commonest Creatinine Clearances determination, built on Cockcroft-Gault Equation ${ }^{[6]}$.

Cockcroft-Gault Formula: Cockcroft-Gault $\mathrm{CrCl}=$ [(140-age) $\mathrm{x}(\mathrm{Wt}$ in $\mathrm{kg}) \times(0.85$ if females $)] /(72 \times \mathrm{Cr})^{[7]}$.

\section{All the cases were subjected to the following:}

History taking, full clinical examination including vital signs (BP, breathing rate, pulse, and temp.) and signs of hypervolemia as congested neck veins, fine basal crepitation, and lower limb edema.

Routine lab examinations (CBC, Urea, Creatinine, RBS, Calcium, Phosphorus and assessment of plasma levels of BNP), chest radiograph, as a reference for the presence of pulmonary congestion, echocardiography for both systolic and diastolic function assessment and lung U/S (US examinations of the anterolateral chest will be done with longitudinal scans of the right and left hemi thoraces, from the $2^{\text {nd }}$ to the $4^{\text {th }}$ (on the right-side to the $5^{\text {th }}$ intercostal cavity. Lung U/S and predictive assessment cases with more than 3 B-lines had a 4-time rise in the opportunity of hospital stay because of HF or of all causes of mortality. Lung ultrasound and therapeutic assessment B-line pattern frequently clear afterward medical therapy and associates with other factors, like radiological and clinical scores of the levels of congestion and BNP.

IVC-US: US investigation was performed in the right paramedian longitudinal level via a $5 \mathrm{MHz}$ convex probe instrument.

\section{Ethical approval:}

The study was approved by The Local Ethics Board of Al-Azhar University and an informed written consent was taken from each participant in the study after explanation of the study design. This work has been carried out in accordance with the Code of Ethics of the World Medical Association (Declaration of Helsinki) for studies involving humans.

\section{Statistical analysis}

The collected data were introduced and analyzed by SPSS V20 for data processing and statistic using an IBM-based computer system.
Quantitative data expressed as mean \pm standard deviation (SD) or standard error (SE). Data was analyzed by independent sample, paired $\mathrm{t}$ test and one way, analysis of variance (ANOVA). While qualitative data were expressed as number and percentage and were analyzed by Chi square (X2) test. The correlation was done using a Pearson correlation test.

The receiver operating characteristic (ROC) curve and $95 \%$ confidence interval (CI) was performed to determine cutoff values for the studied biomarkers. Sensitivity, specificity, positive predictive value (PPV) and negative predictive value (NPV) were determined. $P$ value was considered significant if $<0.05$ and highly significant if $<0.001$.

\section{RESULTS}

The mean age of the studied group was $63.34 \pm$ 7.41 with a range of $49-80$ years. There were $32(64 \%)$ males and $18(36 \%)$ females (Table 1$)$.

Table (1): Distribution of studied cases according to IVC index measurements

\begin{tabular}{|lc|}
\hline & $\begin{array}{c}\text { Cases } \\
(\mathbf{N}=50)\end{array}$ \\
\hline IVC Index Measurements & \\
min IVCD & $1.36 \pm 0.52$ \\
max IVCD & $2.04 \pm 0.42$ \\
IVC-CI & $34.94 \pm 16.65$ \\
\hline
\end{tabular}

The mean min IVCD was $1.36 \pm 0.52$, the mean max IVCD was $2.04 \pm 0.42$ and the mean IVC-CI was 34.94 \pm 16.65 (Table 1).where?

Table (2): Distribution of studied cases according to ultrasound -define 2 columns

\begin{tabular}{|lcc|}
\hline \multicolumn{3}{|c|}{ Cases $(\mathbf{N}=\mathbf{5 0})$} \\
US & number & Percentage \\
Consolidation & 18 & 36.0 \\
Right effusion & 20 & 40.0 \\
B lines & \multicolumn{2}{|}{} \\
$\leq \mathbf{3}$ & 13 & 26.0 \\
$>\mathbf{3}$ & 37 & 74.0 \\
Range & \multicolumn{2}{c}{$0-49$} \\
Median (IQR) & $12(3.25-20.75)$ \\
\hline
\end{tabular}

There were $18(36 \%)$ with consolidation, $20(40 \%)$ with right effusion and there were $13(26 \%)$ with B lines less than 3 with median B lines was 12 with interquartile range of 3.25-20.75 and range of 0-49 (Table 2). 
Table (3): Relation between IVC-CI and Dyspnea Class

\begin{tabular}{|c|c|c|c|c|c|c|c|c|}
\hline \multirow[b]{3}{*}{ Dyspnea Class } & \multicolumn{6}{|c|}{ IVC-CI } & \multirow[t]{3}{*}{ Test } & \multirow[t]{3}{*}{$\mathbf{p}$} \\
\hline & \multicolumn{2}{|c|}{$\begin{array}{c}>50 \\
(\mathrm{n}=13)\end{array}$} & \multicolumn{2}{|c|}{$\begin{array}{c}30-50 \\
(\mathrm{n}=17)\end{array}$} & \multicolumn{2}{|c|}{$\begin{array}{c}<30 \\
(\mathrm{n}=20)\end{array}$} & & \\
\hline & No. & $\%$ & No. & $\%$ & No. & $\%$ & & \\
\hline I & 10 & 76.9 & 0 & 0.0 & 0 & 0.0 & $\chi^{2}=69.3$ & $<0.001^{*}$ \\
\hline II & 3 & 23.1 & 16 & 94.1 & 2 & 10.0 & 03 & \\
\hline III & 0 & 0.0 & 1 & 5.9 & 14 & 70.0 & & \\
\hline IV & 0 & 0.0 & 0 & 0.0 & 4 & 20.0 & & \\
\hline
\end{tabular}

$\chi^{2}$ : Chi-square test

$\mathrm{p}$ : p-value for comparison among the study groups

*: Statistical significance at $\mathrm{p} \leq 0.05$

A highly significant association was found among IVC-CI and dyspnea class (Table 3).

Table (4): Association among IVC-CI and Echo

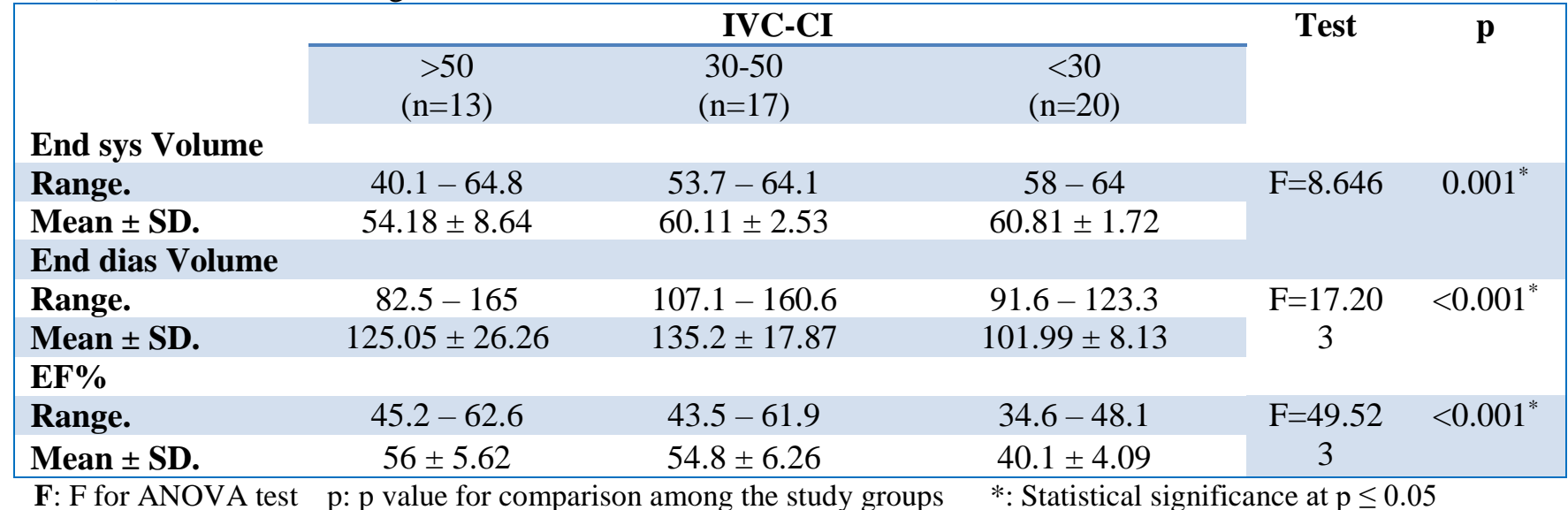

This table shows that there was high statistically significant relation between IVCCI and ECHO (Table 4).

A highly significant association was found among IVC-CI and B lines (Table 5).

Table (5): Relation between IVC-CI and B Lines

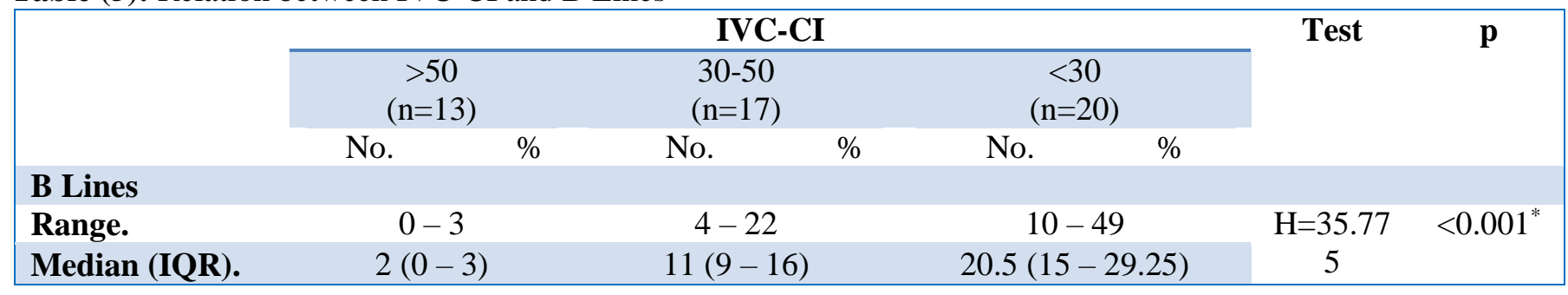

Using B Lines, it was shown that above 32.5, it can discriminate between class IV (severe) and other classes with area under curve of 1.00 , level of sensitivity $100 \%$, specificity $100 \%$, PPV $100 \%$, NPV 100\%, and accuracy $100 \%$ (Figure 1). 


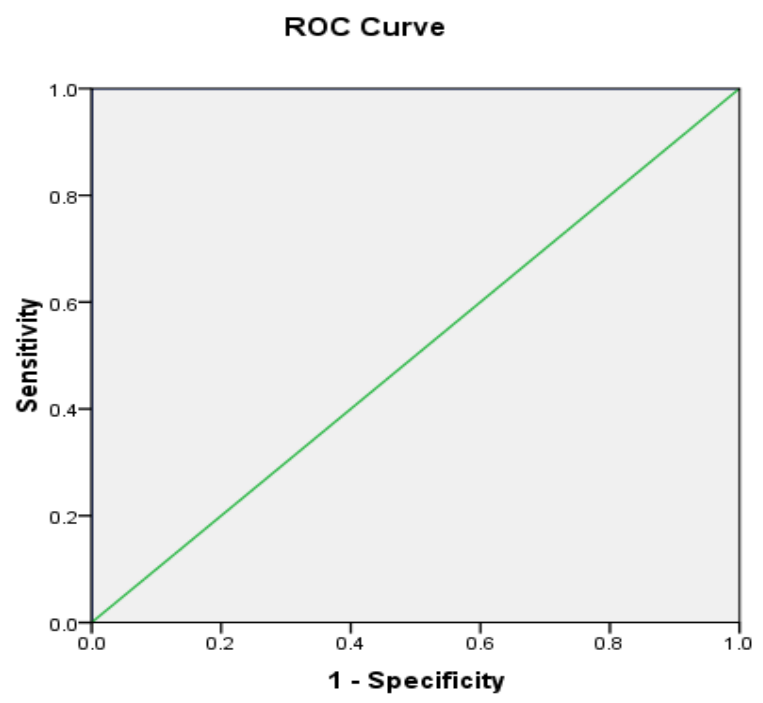

Fig (1): Roc curve analysis for the use of B Lines to discriminate between Class IV (severe) and other classes

\section{DISCUSSION}

Heart failure (HF) is a widespread chronic disorder that is the main reason for hospital stay between cases of ages $>65$ years ${ }^{[8]}$. ADHF, marked by volume excess and secondary congestive symptoms, is the main reason for HF-cases admissions. Knowing outpatients' volume status to regulate treatments and to prevent decompensation is challenging. Physical examinations and radiologic results have a restricted value for determining the volume grade ${ }^{[9]}$.

In our study the majority of patients were males (64\%). This disagrees with Curbelo et al. ${ }^{[10]}$ who showed that the majority was females (58.9\%). Besides, Nagdev et al. ${ }^{[11]}$ reported that $60 \%$ were women. The mean age in our population was $63.34 \pm 7.41$ with range of 49-80 years. It is comparable with corresponding values from other studies conducted by Miller $\boldsymbol{e t}$ al. ${ }^{[12]}$

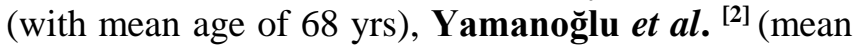
age of 72.8 yrs) and Curbelo et al. ${ }^{[10]}$ reported high average age of $84.3 \pm 6.4$ years).

In our patients, there were 17 (34\%) non-smoker, $5(10 \%)$ mild smokers, 14 (28\%) moderate smokers, and 14 (28\%) severe smokers. While, Kamimura et al. ${ }^{[13]}$ found that $12 \%$ were current smokers, $18 \%$ were former smokers, and $70 \%$ were never smokers.

According to BMI in our population, there were 15 (30\%) with mild BMI, 29 (58\%) with moderate BMI, and $6(12 \%)$ with severe BMI. Parissis et al. [14] reported that overweight/obese cases accounted for $75.7 \%$ of cases and had worse New York Heart Association class $(\mathrm{P}$ value $<0.001)$ and elevated admissions systolic BP (P value <0.001).

In $\mathrm{HF}$, principally in dilated cardiomyopathy, $\mathrm{EF}$ may be very small as SV reduces and EDV rises, in severe $\mathrm{HF}, \mathrm{EF} \leq 20 \%$. EF is frequently utilized as a clinical index to assess the inotropic heart condition. But it is significant to notice that there are situations in which EF could be ordinary, yet the ventricle is in failure. One instance is diastolic dysfunction resulted from hypertrophy in which filling is reduced owing to low ventricular compliances (i.e., "stiff" ventricle) and stroke volume is consequently decreased. In this circumstance, SV, as well as, EDV may be decreased such that EF doesn't alter noticeably. For this cause, low EFs are commonly accompanied by systolic dysfunctions rather than diastolic dysfunctions ${ }^{[15]}$.

In our study, we found that the mean ESV was $58.85 \pm 5.42$, the mean EDV was $119.28 \pm 22.75$ and the mean EF\% was $49.23 \pm 9.17$. Kerkhof et al. ${ }^{[16]}$ examined the current criticism concerning $\mathrm{EF}$, and defined a novel avenue to mark ventricular functions within the uniting basis of cardiac input/output volumes regulations. This method connects end-systolic volumes (ESV) to end-diastolic volumes (EDV) and derives for a sub-group similar in $\mathrm{pEF}$ criteria a separate pattern in the ESV-EDV domain. In cases with $\mathrm{pEF}(\mathrm{n}=34)$, an obvious variance $(\mathrm{P}$ value $<0.0004)$ in the slope of the regressing line for ESV vs. EDV was established in comparison with controls with EF < 50\% ( $\mathrm{n}=29)$. Our results are supported by Curbelo et al. ${ }^{[10]}$ who reported that HF with decreased EF was existing in $14.8 \%$ of cases. And Kerkhof et al. ${ }^{[17]}$ reported that in all patients, ESV arises as the main constituent of EF, with lesser (P-value < 0.0001) influence of EDV. The association for EF vs. ESV is non-linear (P-value < 0.0001 ), and analogous for all genders. A linear method can be insufficient and produce erroneous statistic results when comparing subgroups of cases.

In the current work, the mean minimum IVCD was $1.36 \pm 0.52$, the mean maximum IVCD was $2.04 \pm 0.42$ and the mean IVC-CI was $34.94 \pm 16.65$. According to the US there were $18(36 \%)$ with consolidation, 20 (40\%) with right effusion and 13 (26\%) with B lines less than 3 with median B lines was 12 with interquartile range of 3.25-20.75 and range of 0-49 respectively. Curbelo et al. ${ }^{[10]}$ showed that as regards IVC collapsibility, $24.2 \%$ existing with IVC-CI $>50 \%$ and $38.9 \%$ with IVC-CI $30 \%$ to $50 \%$; and $36.8 \%$ had IVC- 
CI $<30 \%$. De Vecchis et al. ${ }^{[18]}$ found that multi-variate prognosticators of elevated congestion score were jugular venous distension (HR: 13, 38 95\% CI: 2, 13 84 p-value $=0,0059)$ and rattles (HR: $1195 \%$ CI: 1, 45 - 83, 8 p-value $=0,0213)$. IVC-CI $\leq 15 \%$ was continuously accompanying with elevated congestion scores at the $2^{\text {nd }}$ visit, but IVC-CI $\leq 15 \%$ was unsuccessful to expect an elevated congestion score at the $2^{\text {nd }}$ visit.

Concerning US evaluations of the IVC collapsibility index in congestive HF-cases, De Vecchis et al. ${ }^{\left[{ }^{[18]}\right.}$ reported that multi-variate prognosticators of ARD were a lower basal-eGFR (HR: 0.82 CI: 0.72-0.94 $\mathrm{p}$-value $=0.0045$ ) and intra-venous furosemide every day mean dosage greater than $80 \mathrm{mg}$ (HR: $48.62 \mathrm{CI}$ : $1.62-3841.5 \mathrm{p}$-value $=0.043$ ). A very significant positive association was revealed among IVC-CI at admittance $\leq 15 \%$ and basal eGFR $(\mathrm{r}=0.96 \mathrm{p}$ value $<0.0001)$, while a negative association with eGFR was found in the IVCCI maximum $(>40 \%)$ range $(\mathrm{r}=-0.696 \mathrm{p}$ value $=0.0013)$. Moreover, the category with basal IVC-CI > 40\% revealed an elevated rate of ARD in comparison with that with basal IVC-CI 16-40\% ( $\mathrm{p}$ value < 0.05 ).

Yamanoğlu et al. ${ }^{[2]}$ reported that the IVCD determined with B-mode throughout inspirations (Bmode I) was the most effective technique for discriminations between the two groups. B-mode I values $>0.9 \mathrm{~cm}$ expected dyspnea of cardiac origin with a sensitivity of 84.4 percent and a specificity of 92.9 percent (+LR: 11.8, LR: 0.16).

In the current work, a highly significant association was found among IVC-CI and Dyspnea Class. De Vecchis et al. ${ }^{[18]}$ found that a lower $(\leq 15 \%)$ IVC-CI value showed an elevated chance of representing with a higher congestion score that was $\geq$ 4 signs and/or symptoms of congestion within a range spreading between 0 - 7 in comparison with the other 2 clusters of IVC-CI; but, when assessed by Cox comparative hazard regressions analysis counting 8covariates, the lowest IVC-CI class wasn't established to be an important multi-variate prognosticator of clinical congestions kept over a 60 days follow-up on adjusted oral treatment. All of the cases admitted with IVC-CI $\leq 15 \%$ were revealed to have a clinical image of united right and left HF. This result is seemingly accompanying both higher CPWP and right atrial pressures.

Our results revealed that a highly significant association was found among IVC-CI and Echo. Curbelo et al. ${ }^{[10]}$ disagree with our results in reporting that a nonsignificant association was found among IVC$\mathrm{CI}$ and left ventricular EF (LVEF; coefficient 0.11, Pvalue $=0.315$ ) or eGFR (coefficient 0.12, Pvalue $=0.261$ )

In our study, we found that a highly significant association was found among IVC-CI and B lines and a statistically significant relation between IVC-CI and effusion. Neesse et al. ${ }^{\left[{ }^{[19]}\right.}$ found that the commonest diagnose accompanying with acute dyspnea were: (a) acute coronary condition $(\mathrm{n}=12,21 \%)$, (b) decompensated CHF $(\mathrm{n}=11,20 \%)$, and (c) chronic obstructive pulmonary disorder (COPD) $(\mathrm{n}=10,18 \%)$. Pleural effusion was found in $100 \%$ of CHF, $17 \%$ of critical coronary condition, and $20 \%$ of COPD cases, establishing a highly significant factor in the differential diagnosis (P-value $<0.01)$. The US providing a supportive tool in $\mathrm{n}=38(68 \%)$, and further treatment outcomes were drawn in $n=14(25 \%)$.

In our study, Using B Lines, it was shown that above 32.5, it can discriminate between class IV (severe) and other classes with AUC of 1.00, level of sensitivity $100 \%$, specificity 100 percent, PPV 100 percent, NPV 100 percent, and accuracy 100 percent. Platz et al. ${ }^{[20]}$ showed that for the 4-zone scan technique, the median B line number was 2 (IQR, 1-4) for the pocket devices and 3 (1-5) for the higher-end system $(\mathrm{P}$-value $=0.67)$. For the 8 -zone technique, the median B-line number was 4 (2-7) for the pocket devices and 5 (3-7) for the higher-end system (P-value $=0.18$ ). Higher numbers of B-lines were recognized on the 4- vs 2-sec LUS clips (P-value < .001 for 4-zone, Pvalue $=.001$ for 8 -zone), and on the 6-vs 4-sec LUS clips $(\mathrm{P}$-value $=0.057$ for 4 -zone, $\mathrm{P}$-value $=0.018$ for 8 zone). Msolli et al. ${ }^{[21]}$ found that the diagnosing presentation of B-lines score at a cutoff 15 and B-profile pattern was 88 percent and 82.5 percent for sensitivity, 75 percent and 84 percent for specificity, 80 percent and 85 percent for PPV, 84 percent and 81 percent for NPV respectively. The area under ROC-curve was 0.86 [0.83-0.89] and 0.83 [0.80-0.86], respectively, for Blines scores and B-profile patterns. There was a best matching among inhabitants for the CHF diagnosing via the two scores (kappa $=0.81$ and 0.85 , respectively, for ordinal scale B-lines scores and B-profile patterns). Spevack et al. ${ }^{[22]}$ showed a tendency to significance for the B-lines number where there was a $38 \%$ reduction in the B-lines number with HF-therapy. While Manson $\boldsymbol{e t}$ al. ${ }^{[23]}$ reported that built on the threshold levels of BNP $500 \mathrm{pg} / \mathrm{mL}$, the sensitivity of result two-sided B-lines on US was 33.3 percent ( 95 percent CI: $0.19-0.50$ ), and the specificity was 91.7 percent ( 95 percent CI: 0.730.99). Furthermore, two-sided B-lines were missing in all BNP-cases $<100-\mathrm{pg} / \mathrm{mL}$. And, Staub et al. ${ }^{[24]}$ found that the AUC of ROC-curve of LUS was 0.914 for acute HF. In acutely dyspneic cases, adapted diffuse interstitial condition had a sensitivity of 0.90 (95 percent CI $0.87-0.93$ ) and specificity of 0.93 (95 percent CI 0.91-0.95) for acute HF.

\section{CONCLUSION}

Ultrasound can be utilized as an alternative technique for approximating the intra-vascular volume like the determination of IVCD and the caval index. The usage of PU to evaluate dyspneic cases and those with HF in dissimilar clinical settings raises the specificity, sensitivity, and accuracy of the pulmonary congestion diagnosing and prognosis for HF cases. 
Conflict of interest statement: The authors declared that there were NO conflicts of Interest.

Disclosure: The authors have no financial interest to declare in relation to the content of this article.

Authorship: All authors have a substantial contribution to the article.

\section{REFERENCES}

1. Anderson K (2013): Diagnosing heart failure among acutely dyspneic patients with cardiac, inferior vena cava, and lung ultrasonography. The American journal of emergency medicine, 31(8): 1208-1214.

2. Yamanoğlu A (2015): The role of inferior vena cava diameter in the differential diagnosis of dyspneic patients; best sonographic measurement method? The American journal of emergency medicine, 33 (3): 396401.

3. Kenneth D, Alain C, Gerasimos F (2008): ESC Guidelines for the diagnosis and treatment of acute and chronic heart failure, 8: 2395

4. Labovitz A (2010): Focused cardiac ultrasound in the emergent setting: a consensus statement of the American Society of Echocardiography and American College of Emergency Physicians. Journal of the American Society of Echocardiography, 23 (12): 1225-1230.

5. Hebl V, Zakharova M, Canoniero M (2012): Correlation of natriuretic peptides, and inferior vena cava size in patients with congestive heart failure. Vasc Health Risk Manag., 8: 213-8.

6. Morbitzer K , Jordan J, Dehne K et al. (2019): Enhanced renal clearance in patients with hemorrhagic stroke. Critical care medicine, 47 (6): 800-808.

7. Al-Dorzi H, Eissa A , Khan R et al.(2019): Dosing errors of empirical antibiotics in critically ill patients with severe sepsis or septic shock: a prospective observational study. International journal of health sciences, 13 (4): 48.

8. Jackson S, Tong X, King $\mathbf{R}$ et al. (2018): National burden of heart failure events in the United States, 2006 to 2014. Circulation: Heart Failure, 11 (12): e004873.

9. Curbelo J, Rodriguez-Cortes P, Aguilera M, GilMartinez P, Martín D, Suarez F (2019): Comparison between inferior vena cava ultrasound, lung ultrasound, bioelectric impedance analysis, and natriuretic peptides in chronic heart failure. Current medical research and opinion, 35(4): 705-713.

10. Curbelo J, Aguilera M, Rodriguez-Cortes P, GilMartinez P, Suarez FC (2018): Usefulness of inferior vena cava ultrasonography in outpatients with chronic heart failure. Clinical cardiology, 41 (4): 510-517.

11. Nagdev A, Merchant $R$, Tirado-Gonzalez A et al. (2010): Emergency department bedside ultrasonographic measurement of the caval index for noninvasive determination of low central venous pressure. Annals of emergency medicine, 55 (3): 290295.
12. Miller B, Sen A, Strote S (2012): Inferior vena cava assessment in the bedside diagnosis of acute heart failure. Am J Emerg Med., 30: 778-83.

13. Kamimura D, Cain L, Mentz R et al. (2018): Cigarette smoking and incident heart failure: insights from the Jackson Heart Study. Circulation, 137 (24): 2572-2582.

14. Parissis J, Farmakis D, Kadoglou N et al. (2016): Body mass index in acute heart failure: association with clinical profile, therapeutic management and in-hospital outcome. European journal of heart failure, 18 (3): 298305.

15. Vriz O, Elmula F, Antonini-Canterin F (2021): Noninvasive Assessment of Ventricular-Arterial Coupling in Heart Failure. Heart Failure Clinics, 17 (2): 245-254.

16. Kerkhof P, Yasha J, Li J, Heyndrickx G (2013): Left ventricular volume regulation in heart failure with preserved ejection fraction. Physiological reports, 1(2):e0007

17. Kerkhof $P$, van de Ven $P$, Yoo B et al.(2018): Ejection fraction as related to basic components in the left and right ventricular volume domains. International journal of cardiology, 255: 105-110.

18. De Vecchis R, Ciccarelli A, Ariano C (2012): Inferior Vena Cava collapsibility and heart failure signs and symptoms: new insights about possible links. Arquivos brasileiros de cardiologia, 98 (6): 544-552.

19. Neesse A, Jerrentrup A, Hoffmann S, Sattler A, Görg C, Kill C et al. (2012): Prehospital chest emergency sonography trial in Germany: a prospective study. European Journal of Emergency Medicine, 19 (3): 161166.

20. Platz E, Pivetta E, Merz AA, Peck J, Rivero J, Cheng S (2015): Impact of device selection and clip duration on lung ultrasound assessment in patients with heart failure. The American journal of emergency medicine, 33 (11): 1552-1556.

21. Msolli M, Sekma A, Marzouk M et al. (2021): Bedside lung ultrasonography by emergency department residents as an aid for identifying heart failure in patients with acute dyspnea after a 2 -h training course. The Ultrasound Journal, 13 (1): 1-8.

22. Spevack R, Al Shukairi M, Jayaraman D, Dankoff J, Rudski L, Lipes J (2017): Serial lung and IVC ultrasound in the assessment of congestive heart failure. Critical ultrasound journal, 9 (1): 1-7.

23. Manson W, Bonz J, Carmody $\mathrm{K}$ et al. (2011): Identification of sonographic B-lines with linear transducer predicts elevated B-type natriuretic peptide level. Western Journal of Emergency Medicine, 12 (1): 102.

24. Staub L, Biscaro R, Kaszubowski E, Maurici $\mathbf{R}$ (2019): Lung ultrasound for the emergency diagnosis of pneumonia, acute heart failure, and exacerbations of chronic obstructive pulmonary disease/asthma in adults: a systematic review and meta-analysis. The Journal of emergency medicine, 56 (1): 53-69. 